\title{
The time-dependent evolutionary rate of mitochondrial DNA in small mammals inferred from biogeographic calibration points with reference to the late Quaternary environmental changes in the Japanese archipelago
}

\author{
Hitoshi SuZuKI ${ }^{1 *}$ \\ ${ }^{1}$ Graduate School of Environmental Science, Hokkaido University, North 10, West 5, Sapporo 060-0810, Japan
}

Received 17 October 2020; accepted 1 December 2020

\begin{abstract}
Mitochondrial DNA (mtDNA) sequences have long been the most popular marker for assessing phylogenetic relationships and uncovering population dynamics. However, the mechanism of the nucleotide substitution rate of mtDNA remains unclear. While the evolutionary rate over tens of thousands of years is thought to be time dependent, the overall picture is not fully understood. This article presents recent achievements related to the time-dependent evolutionary rate of mtDNA in small rodents in the Japanese archipelago. The method focuses on rapid expansion events during the late Quaternary, during which there was a prolonged severe cold period and repeated abrupt warm periods, providing multiple calibration points. The global sea level fluctuation and migration to islands help to specify the calibration points. For calibration points at $11000,15000,53000$, and 130000 years ago, the evolutionary rates were approximately $0.11,0.11,0.047$, and 0.029 substitutions/site/million years, respectively, in the mitochondrial cytochrome $b$ gene $(C y t b)$. Applying the higher rate to assess the evolutionary history of the commensal house mouse (Mus musculus) and complete mitochondrial genome sequences $(\sim 16000 \mathrm{bp}$ ) allowed us to trace prehistoric human culture development based on millet and rice agriculture. The pattern of time-dependent evolutionary rates presented here is likely applicable to other small rodents. The Japanese archipelago is ideal for assessing evolutionary rates with biogeographic calibration points in the late Quaternary in species with multiple genetically distinct local populations.
\end{abstract}

Key words: land bridge, mitochondrial DNA, murine rodents, Quaternary glacial maximum, timedependent evolutionary rate

\section{Introduction}

Mitochondrial DNA (mtDNA) has long been the phylogenetic marker most commonly used to assess species relationships, genetic structure, and population dynamics (Brown et al., 1979; Avise, 1986; Irwin et al., 1991). The evolutionary rate of this marker proposed more than 40 years ago is $0.01-0.02$ substitutions/site/million years (myr) in mammals (Brown et al., 1979; Wilson et al., 1985). Since then, however, no further consensus has been made on more accurate evolutionary rates. Ho and Larson (2006) proposed that the evolutionary rate of mtDNA has changed over time, namely the substitution rate is significantly higher than in older divergences (see also Penny, 2005). However, the rates currently used for the recent divergences differ among researchers, ranging from 0.11 (e.g. Suzuki et al., 2015) to 0.4 (e.g.

\footnotetext{
* Correspondence to: Hitoshi Suzuki, Graduate School of Environmental Science, Hokkaido University, North 10, West 5, Sapporo 060-0810, Japan.

E-mail: htsuzuki@ees.hokudai.ac.jp

Published online 31 March 2021

in J-STAGE (www.jstage.jst.go.jp) DOI: 10.1537/ase.201201
}

Rajabi-Maham et al., 2008; Herman and Searle, 2011; García-Rodríguez et al., 2018) substitutions/site/myr in rodents and around 0.05 in humans (protein-coding region; Henn et al., 2009). Here, I propose a substitution rate curve that shows a time-dependent tendency by utilizing the nucleotide substitution rate of mtDNA evaluated from biogeographic evidence.

I focus on historical events during the last 150000 years, when much of the planet was subject to rapid massive climate fluctuations (Brown et al., 2007). Specifically, a prolonged glacial period during the late Quaternary induced extreme population reductions, whereas abrupt rapid warmings triggered sudden population increases. Sudden population expansion can be detected by drawing a star-shaped network of mtDNA sequences and using biogeographic calibration points, which allow estimation of the evolutionary rate. Another way to find biogeographic calibration points is by addressing the dispersal events associated with the construction and destruction of land bridges facilitating migration between land masses separated by deep sea (i.e. at least $120 \mathrm{~m}$ depth; Japan Association for Quaternary Research, 1987). While the sea level rose during periods of global warming (Lambeck et al., 2014), the land bridges are 
thought to have remained during the initial warming, allowing dispersal among what are now island masses. Bottlenecks are also caused by migration, as a founder effect, providing an opportunity to set a calibration point (e.g. Henn et al., 2009). For example, the house mouse (Mus musculus) migrated to the Japanese archipelago with humans around 3000 years ago along with the introduction of early paddy rice cultivation in the Yayoi period (Crawford and Lee, 2003; Fuller, 2011), yielding a star-shaped network in mtDNA analyses (Kuwayama et al., 2017; Li et al., 2020b).

\section{Characterization of rapid expansion events in small rodents from Japan}

\section{Population dynamics in small mammals inferred from mtDNA variation}

Phylogenetic analysis, such as the construction of a maximum likelihood tree and bootstrap analysis, helps us to specify clusters of mtDNA sequences. Network construction with mtDNA sequences sometimes exemplifies a cluster with a star-shaped structure, as mentioned above. The distribution of pairwise differences or mismatch distribution gives a unimodal pattern that is also indicative of rapid expansion (Slatkin and Hudson, 1991; Rogers and Harpending, 1992). Significantly negative values in neutrality tests, such as Tajima's $D$ and Fu's $F_{\mathrm{S}}$, confirm rapid expansion events.

The peak (mode) of the mismatch distribution is defined as $\tau$, which is a statistical value proportional to the time since expansion. From the $\tau$ value, the time in generations $(t)$ since expansion started is calculated from the formula $t=\tau / 2 u$, where $u$ is the mutation rate per generation for the entire haplotype (Rogers and Harpending, 1992). Here, $u=\mu k g$, where $\mu$ is the evolutionary rate (substitutions/site/years), $k$ is the sequence length of the haplotype, and $g$ is the generation time in years. The time in years $(T)$ since expansion can be estimated using the formula $T=\operatorname{tg}=\tau / 2 \mu \mathrm{k}$. The value of $\tau$ is an average value obtained by comparing a large number of sequences; thus, the value is trustworthy. Assigning historic time points $(T)$ for the start of expansion, the evolutionary rate in years $(\mu)$ can be calculated with the formula $\mu=d / 2 T$, where $d$ is the genetic distance $(d=\tau / k)$.

Our previous study addressed mtDNA cytochrome $b$ gene $(C y t b)$ sequences, which is the most popular mtDNA gene for phylogenetic inference in mammals. Our study of the $C y t b$ gene sequences (1140 bp) of the large Japanese wood mouse Apodemus speciosus (Suzuki et al., 2015) detected an apparent signal for a population expansion event in the sequence clusters for Hokkaido and Honshu/Shikoku/Kyushu with $\tau=2.7$ and 9.2, respectively. Then, we measured the $\tau$ value excluding the haplotypes from islands associated with the main islands of Honshu and Kyushu and obtained a new $\tau$ of 8.5 (Honda et al., 2019). In the lesser Japanese wood mouse Apodemus argenteus, the Hokkaido population shows mtDNA diversity that indicated a recent rapid expansion, with a $\tau$ value of 2.6 (Suzuki et al., 2015). By contrast, the sequences from the three main islands of Honshu, Shiko$\mathrm{ku}$, and Kyushu and their adjacent islands (Sado, Oki, Yakushima, etc.; Figure 1A) showed five clusters with expansion signals (Hanazaki et al., 2017), in which the $\tau$ values could be categorized into three groups based on the values
3.9, 5.7, and 7.8-8.5. The Japanese giant flying squirrel Petaurista leucogenys, a rodent inhabiting broadleaf forests, had two clusters with expansion signals in $C y t b$ sequences with $\tau$ values of 3.9 and 8.3 (Oshida et al., 2009). Similarly, in a study of grassland-dwelling voles occurring in the Japanese archipelago, we found four clusters with expansion signals from three species: two from Hokkaido (Myodes rufocanus and Myodes rutilus) and one from Honshu/Shikoku/ Kyushu (Myodes smithii). The $\tau$ values obtained formed two groups: 4.1 and 5.8-6.3.

Overall, the $\tau$ values observed in the Cytb sequences of small mammals from the Japanese archipelago can be roughly attributed to three groups of historic time points based on $\tau$ values (Cytb, $1140 \mathrm{bp}$ ): Groups I, II, and III for 2.6-4.1, 5.8-6.3, and 7.8-8.5, respectively. A trend similar to these $\tau$ values can be seen in the mtDNA sequences of Eurasian populations of $M$. rufocanus (Abramson et al., 2012; Honda et al., 2019). The $\tau$ values representing Group I can be subdivided into groups Ia $(\tau=2.6-2.7)$ and $\mathrm{Ib}$ ( $\tau=3.9-4.1$ ) (Hanazaki et al., 2017; Honda et al., 2019).

\section{Linking population events to turning points in the Quaternary environment}

How can one link the four historic expansion events to specific geological events? Since the species considered (wood mice, voles, and flying squirrels) are non-commensal, these rapid expansion events could be attributed to environmental recovery from the harsh glacial periods in the Quaternary, especially after the glacial maxima that recurred every 100000 years, as many researchers have noted (e.g. Hewitt, 2004; Oshida et al., 2009; Herman and Searle, 2011).

The turning points in the late Quaternary environment from cold to warm stages are the best occasions to consider as the starting points of population expansions after a bottleneck and subsequent sudden population growth. The transitions from marine isotope stage (MIS) 2 to MIS 1 (10000 15000 years ago) and from MIS 6 to MIS 5 (130000 years ago) are the most likely starts of rapid expansion events (Figure 1B). However, the timing of the expansion event of the remaining group may be difficult to precisely determine. Hanazaki et al. (2017) suggested that the transition from MIS 4 to MIS 3 (the early MIS 3), around 53000 years ago when the climate became substantially warmer in the last ice age (Helmens et al., 2009; Helmens and Engels, 2010; Weber et al., 2018), is a candidate turning point.

The vegetation reconstruction over the past 150000 years provides insight into this assignment (Figure 1C; Igarashi and Oba, 2006). Fossil pollen analyses including Quercus species use the $T p$ index, the ratio of the pollen abundance of cool-temperate broadleaf trees to the sum of that of cool-temperate broadleaf plants and subarctic conifers (Igarashi and Oba, 2006). Several peaks of recovery of broadleaf plants have been detected, including those around 15000, 50000-60000, and 130000 years ago (Igarashi and Oba, 2006). These effectively explain the expansion events of acorn-dependent wood mice and large flying squirrels (Hanazaki et al., 2017). By contrast, voles are typical herbivorous animals that were affected by early MIS 3, which corresponds to a period of grassland expansion (Honda et 
A

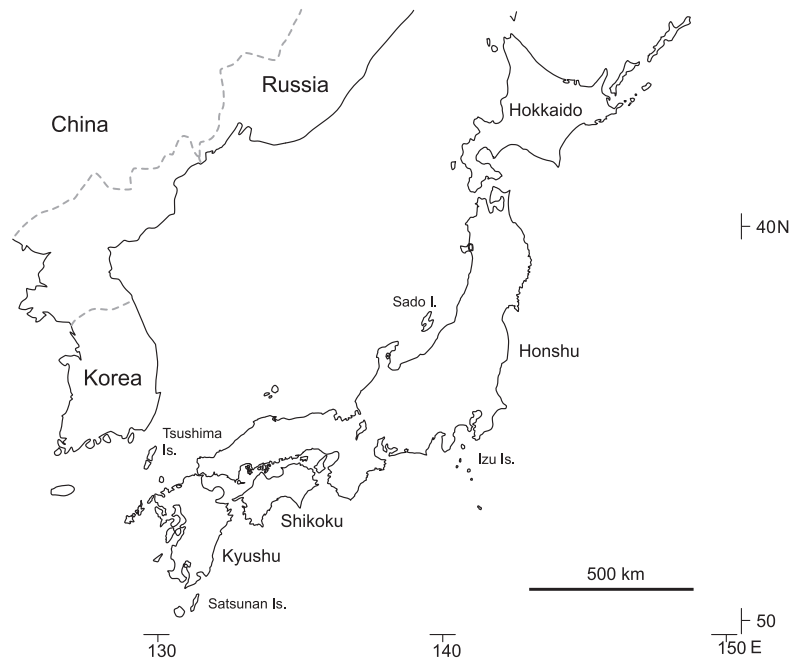

B

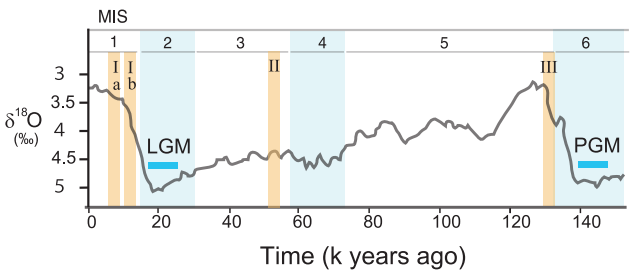

D

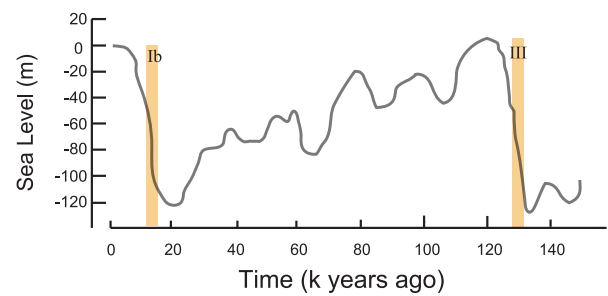

$\mathrm{F}$

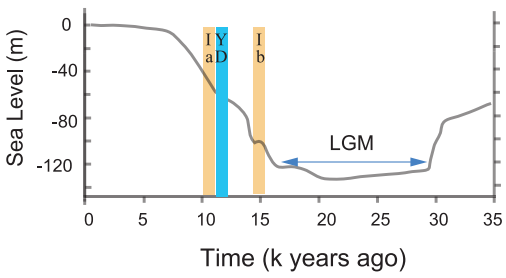

C

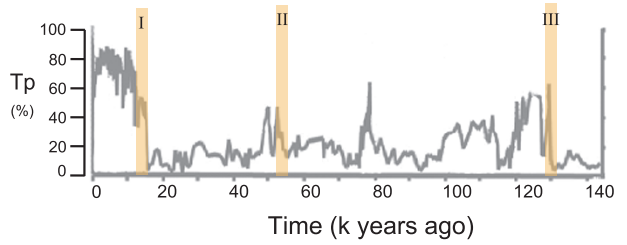

E

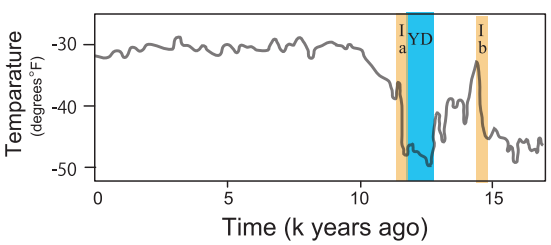

Figure 1. The possible influence of Paleo-environmental fluctuations on the population dynamics of terrestrial animals. (A) A map of the Japanese archipelago showing the four main islands of Hokkaido, Honshu, Shikoku, and Kyushu, along with the associated islands of Sado, Izu, Tsushima, and Satsunan. These surrounding islands are separated from their respective nearby major islands, as well as from Hokkaido and Honshu, by deep sea (i.e., $-120 \mathrm{~m}$ ). (B) The marine oxygen isotope curve over the last 150000 years, adapted from Lisiecki and Raymo (2005), showing the marine isotope stages (MIS). The approximate periods of the last glacial maximum (LGM) and penultimate glacial maximum (PGM) are indicated with horizontal bars. The four critical time points for population expansion events are marked with vertical bars, called Ia, Ib, II, and III here. (C) Inferred climatic changes over 144000 years based on high-resolution pollen analysis of a core from the northwest Pacific off the coast of central Japan (Igarashi and Oba, 2006). The Tp value is the ratio of the pollen abundance of cool-temperate broadleaf trees to the sum of that of cooltemperate broadleaf plants and subarctic conifers (defined by Igarashi and Oba, 2006). (D) The sea level change over the last 140000 years (Lambeck et al., 2014), highlighting the two critical time points (Ib and III) when the temperature became warm and the sea levels were very shallow, allowing gene flow between remote islands via land bridges. (E) A global temperature fluctuation since ca. 17000 years ago (source: https:// www.climate.gov/sites/default/files/historictemperaturerecord greenland large.jpg), indicating the critical time points of Ia and Ib and the period of the Younger Dryas (YD). (F) Global sea level change for the last 35000 years, covering LGM and YD (Lambeck et al., 2014; Harrison et al., 2019). 
al., 2019). The importance of the early MIS 3 period for demographic expansion has been noted in grassland voles in North America (Kohli et al., 2015), and in hares in Europe (Fickel et al., 2008). However, an early MIS 3 expansion event has also been posited in a forest-dwelling wood mouse (A. argenteus) from Japan (Hanazaki et al., 2017) and roof rats (Rattus rattus) in Myanmar (Maung Maung Theint et al., 2020).

For the two Group I subgroups of $\tau$ values, Ia and Ib can be assigned to the expansion events immediately after the termination (c. 11000 years ago) of the Younger Dryas (YD) and the onset of the Bølling-Allerød warming (c. 15000 years ago), respectively, although the environmental recovery is latitude dependent and there are some time differences among regions (Igarashi et al., 2012). YD, a global cold reversal event, is thought to have affected the population dynamics of terrestrial animals (Herman and Searle, 2011; Suzuki et al., 2015).

The assignment of Stages Ib and III to the time periods near the last glacial maximum (LGM) and penultimate (PGM) glacial maximum is congruent with the fact that the haplogroups corresponding to these stages contain members that found refuge on remote islands, such as Sado and Tsushima Islands, currently separated by deep sea. This implies that the expansion events at Stages Ib and III involved migration over land bridges across deep straits at a time when the sea level was low, about $-100 \mathrm{~m}$ (Figure 1D, F).

From these considerations, calibration points can be set for the four stages: Ia, Ib, II, and III, respectively (see Figure 1B). Previously, we obtained representative $\tau$ values for the mitochondrial Cytb sequences (1140 bp) corresponding to the four stages (Suzuki et al., 2015; Hanazaki et al., 2017; Honda et al., 2019). If we use $\tau$ values for the four stages of $2.7,3.9,5.7$, and 8.5 , respectively, the evolutionary rates $(\mu)$ of $C y t b$ are estimated to be $0.11,0.11,0.047$, and 0.029 substitution/site/myr (Figure 2); the first two points have identical evolutionary rates. Notably, this curve has two phases, which I call Phases I (high substitution rates) and II (low substitution rates).

Contrary to initial expectations, the evolutionary patterns of the time dependency were similar among small rodent species and other animals. A study of the Eurasian jay (Garrulus glandarius) from Honshu and Kyushu, Japan detected an expansion signal in the Cytb sequences (1143 bp) with a $\tau$ value of 1.8 (Aoki et al., 2018). If we set the calibration point as 11000 years ago (around the end of YD), the calculated evolutionary rate is 0.072 substitutions/site/myr. In the arthropod Ligidium japonicum, mitochondrial cytochrome c oxidase I gene (COI) sequences (496 bp) from Hokkaido, northern Japan, showed an apparent rapid expansion signal (Harigai et al., 2020). Assuming that the expansion event happened immediately after the end of YD (c. 10000 years ago in Hokkaido; Igarashi et al., 2012), the calculated evolutionary rate of COI is 0.084 substitutions/ site/myr, with a $\tau$ value of 0.83 (Harigai et al., 2020). Therefore, the mtDNA evolutionary rates calculated for divergence on short evolutionarily timescales may not be markedly different among animals including those of vertebrates and invertebrates.

It is possible to estimate the evolutionary rate by considering the divergence of $A$. speciosus on the remote islands of Hokkaido, Sado, Tsushima, Izu, and Satsunan, which are predicted to be have been connected to the main islands of Japan (i.e. Honshu, Shikoku, and Kyushu) via land bridges that arise when the sea level drops every 100000 years (Figure 3A; see Hanazaki et al., 2017). Three calibration points were considered - 140000,250000 , and 350000 years agoallowing the estimation of an evolutionary rate of around 0.03 substitutions/site/myr (Figure 3B, C). The evolutionary rate curve was in good agreement with those derived from the calibration points for the last 130000 years mentioned above (Figure $3 \mathrm{C}$ ). The pattern of the evolutionary rate curve in rodents is similar to that reported for human mtDNA (Henn et al., 2009).

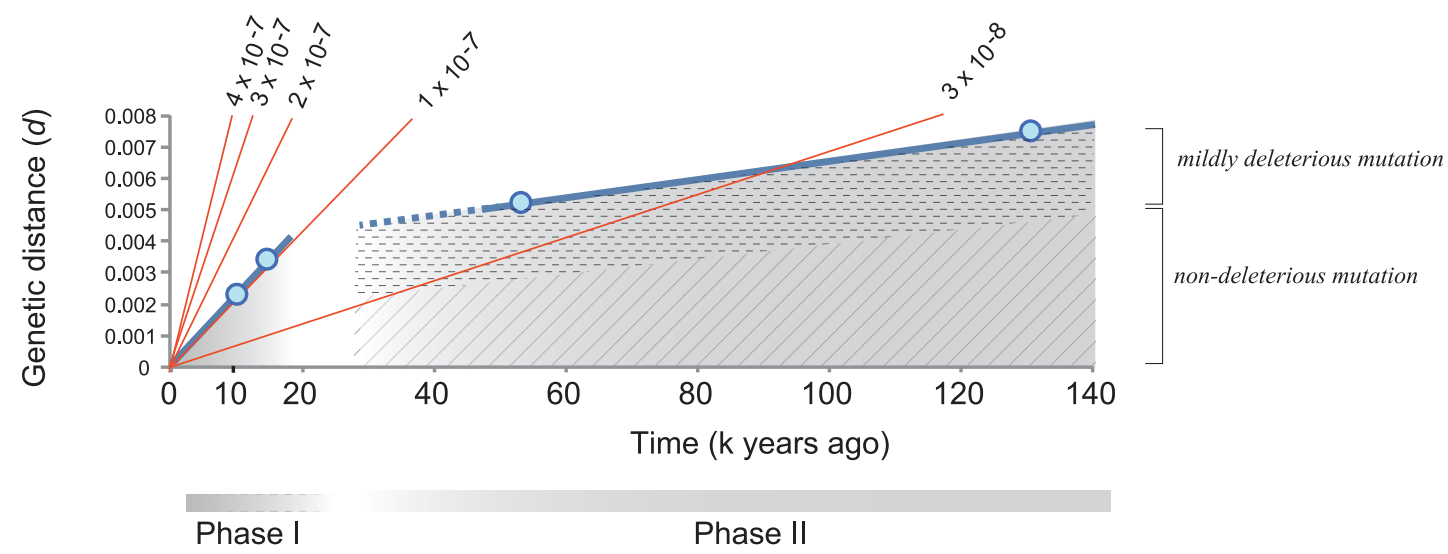

Figure 2. A plot of genetic distances based on rapid expansion events of the Japanese wood mouse species Apodemus speciosus and Apodemus argenteus, tentatively assigning the historic time points $11000,15000,53000$, and 130000 years ago. These points were obtained from the assumption of the association of specific rapid cooling and rapid warming events. The plot comprises two phases, designated Phases I and II. Nucleotide substitutions are considered to be of two types: mildly deleterious and non-deleterious mutations, which are expected to be removed and retained from the sequences, respectively. Five straight lines of the genetic distances with the respective evolutionary rates (substitutions/site/year) are indicated for comparison. 
A

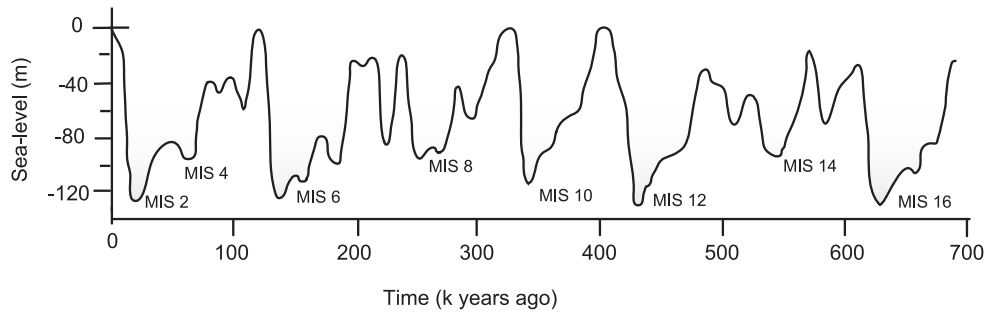

B

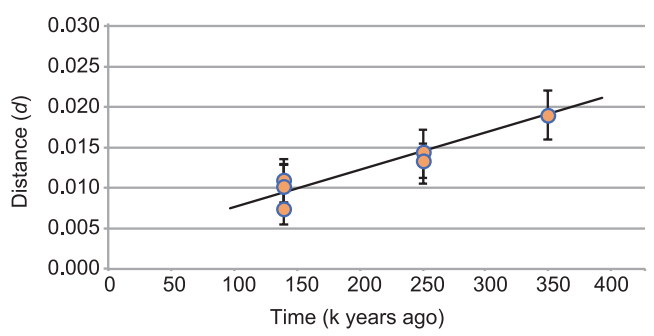

C

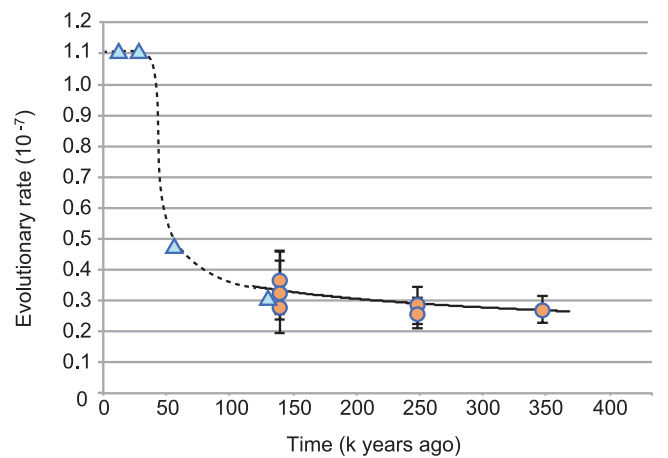

Figure 3. (A) Sea level change during the last 700000 years (Bintanja et al., 2005). (B) p-distances for the genetic divergence (d) of insular lineages of Apodemus speciosus (see Table 1 in Hanazaki et al., 2017) are plotted under the assumption that divergence was associated with the appearance and disappearance of land bridges in a limited time during the glacial maxima. (C) The evolutionary rates predicted in this study are plotted based on the genetic distances shown in B (circles) and Figure 2 (triangles).

\section{Comparison with other mtDNA studies of small rodents}

\section{Concern regarding the highly accelerated evolutionary rates}

There is still a huge range in the rate of mtDNA evolution considered by researchers. When inferring divergence times on shorter timescales based on the mtDNA D-loop (control region) and $C y t b$ in rodent phylogeographic studies, an accelerated evolutionary rate of around 0.4 substitutions/site/ myr (e.g. Rajabi-Maham et al., 2008; Herman and Searle, 2011) is often used; a rate of around 0.1 is used less frequently (e.g. Rajabi-Maham et al., 2008; Suzuki et al., 2013). Here, I would like to point out concerns regarding the higher evolutionary rate.

First, Rajabi-Maham et al. (2008) determined the higher evolutionary rate of mtDNA (0.4 substitutions/site/myr) assuming that the European subspecies of the house mouse, Mus musculus domesticus (DOM), underwent expansion 12000 years ago. However, they presented another option with the expansion starting 60000 years ago using an evolu- tionary rate of 0.1 substitutions/site/myr. I favor the second option, because a range-expansion event has not always triggered a rapid increase in the genetic diversity of mtDNA; instead, it was often associated with ancient divergence in mtDNA. Relatedly, we revealed that a Japanese rodent ( $M$. smithii) acquired genetic variation in mtDNA in ancient times, and shows no sign of having been affected by the relatively recent rapid expansion event, namely the post-LGM event, although it has extended its distribution range to northern Honshu at present (Honda et al., 2019). This may be because the LGM was insufficiently intense to create a bottleneck.

Second, if we draw a curve showing the base substitutions with a high evolutionary rate of 0.4 substitutions/site/myr over the short timescale, it is difficult to connect the two straight lines of Phases I and II, as shown in Figure 2. If the evolutionary rate of Phase I is high (e.g. more than 0.2 substitutions/site/myr), it seems unlikely to accommodate the two genetic distance curves of Phases I and II.

Third, phylogeographic studies using a high evolutionary rate of 0.4 substitutions/site/myr inferred very recent expan- 
sion, over the past few thousand years, and tend to predict that human activity is a major determinant of expansion events, even in wild rodents (e.g. Herman et al., 2017). However, it is difficult to accept the idea that humans influenced the population dynamics of non-commensal animals such as wood mice and voles.

\section{Population dynamics of wood and house mice}

For comparison with Japanese wood mouse species, I examined the mtDNA variation of the European wood mouse Apodemus sylvaticus from Italy and the western Balkans (Clade Ia; Michaux et al., 2003). Using Cytb sequences (949 bp) with no ambiguous sites $(n=16)$ reported in the literature (Michaux et al., 2003; Kryštufek et al., 2012), a median-joining network (Bandelt et al., 1999) was constructed (Figure 4A). This had a star-shaped pattern suggestive of a past rapid expansion event, which was supported by Tajima's $D$ and Fu's $F$ s analyses. This was consistent with the results of a mismatch distribution analysis using Arlequin 3.5.1.2 (Excoffier, 2004; Excoffier and Lischer, 2010) showing a unimodal pattern for both observed and simulated data (Figure 4B, C) and a non-significant sum of the squared deviation and raggedness values (data not shown). The $\tau$ value obtained was 7.20 and the estimated time $(T)$ when the expansion started was 131000 years ago $(T=7.2 / 2 / 949 / 0.29)$, with an evolutionary rate of 0.29 substitutions/site/myr. Notably, the network yielded a small star-shaped cluster of haplotypes from Italy (Cluster Ia-1). Although the number of sequences was small $(n=7)$, the calculated $\tau$ value was 2.1 , implying that the expansion event started 10000 years ago. This suggests that a post-LGM event promoted a rapid expansion somewhere in Italy. Overall, the trend in the occurrence of expansion events was similar to that of the Japanese wood mouse, in which the two expansion events follow the PGM and LGM. Considering this with the results of previous studies (e.g. Michaux et al., 2003; Herman et al., 2017), effort should be made to reconstruct the evolutionary history of the population dynamics of $A$. sylvaticus.

Next, I considered the population dynamics of the European subspecies of $M$. musculus, DOM, in which many researchers, including anthropologists, are interested as a route to better understanding the link between mouse history and prehistoric human activities. I obtained $C y t b$ sequences (958 bp) from databases representing western Europe and adjacent Asian countries, including Turkey and Israel ( $n=107$; Suzuki et al., 2013; Fornuskova et al., 2014), and constructed a median-joining network (Figure 5A). The network was star-shaped and comprised 15 distinct lineages, six of which were characterized as haplogroups (denoted as HG), as reported by Fornuskova et al. (2014), with the starshaped pattern suggestive of past rapid expansion events. The sizes differed among the six haplogroups, suggesting that the expansion events happened at different times during the course of evolution.
A

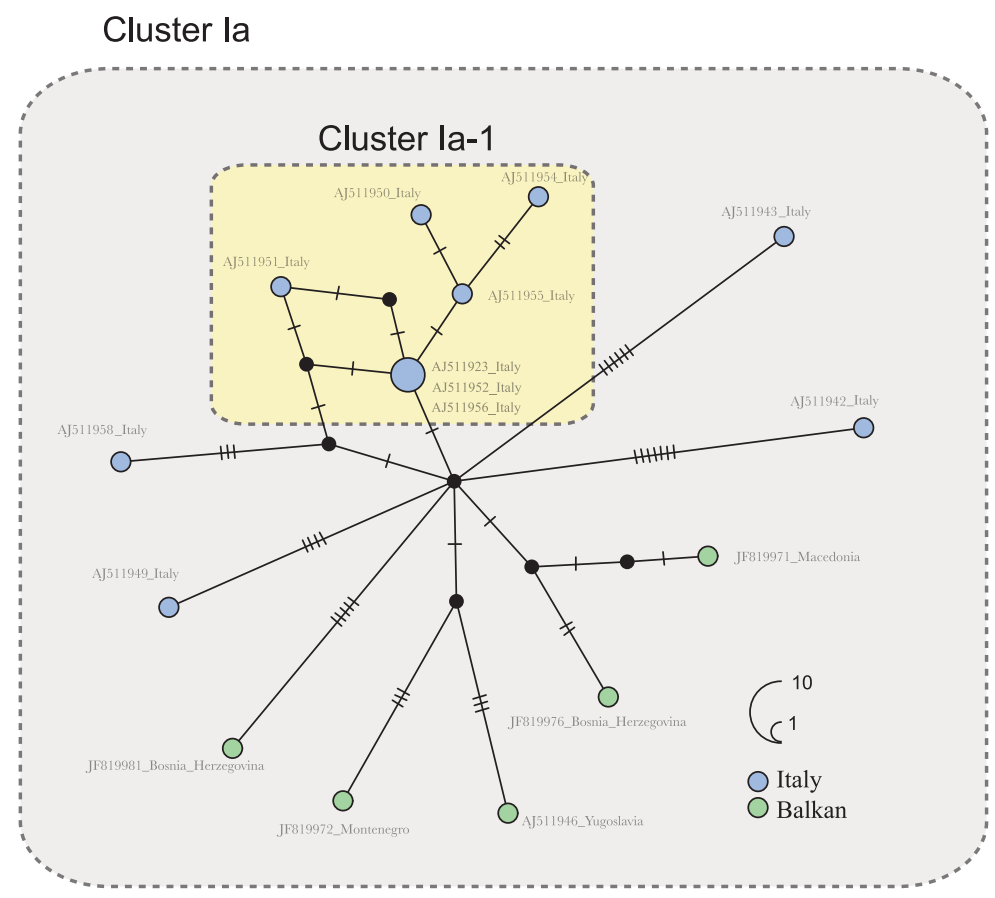

B

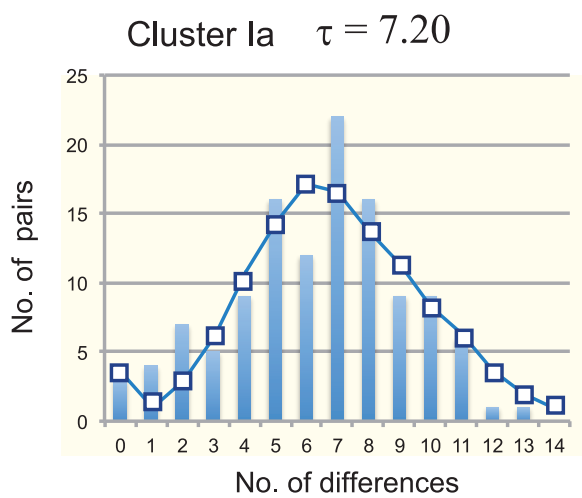

C

Cluster la-1 $\tau=2.10$

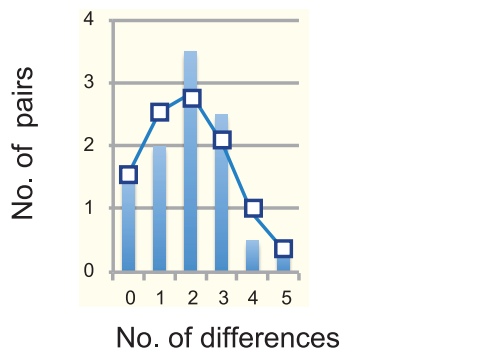

Figure 4. Median-joining networks constructed from (A) Cytb sequences ( $n=16 ; 949 \mathrm{bp}$ ) of Apodemus sylvaticus from Italy and the Balkans. Michaux et al. (2003) called the cluster 'Subclade Ia.' The number of mutations between haplotypes is indicated on the branches. The size of the circles is proportional to the number of samples. The compositions of sample localities are also reflected in each haplotype. Mismatch distributions of the mitochondrial Cytb sequences of Clusters Ia (B) and Ia-1 (C) indicated significant evidence of expansion. Bars indicate the observed frequencies of mutations between haplotypes and the line is the expected frequency under the sudden expansion model. 
A

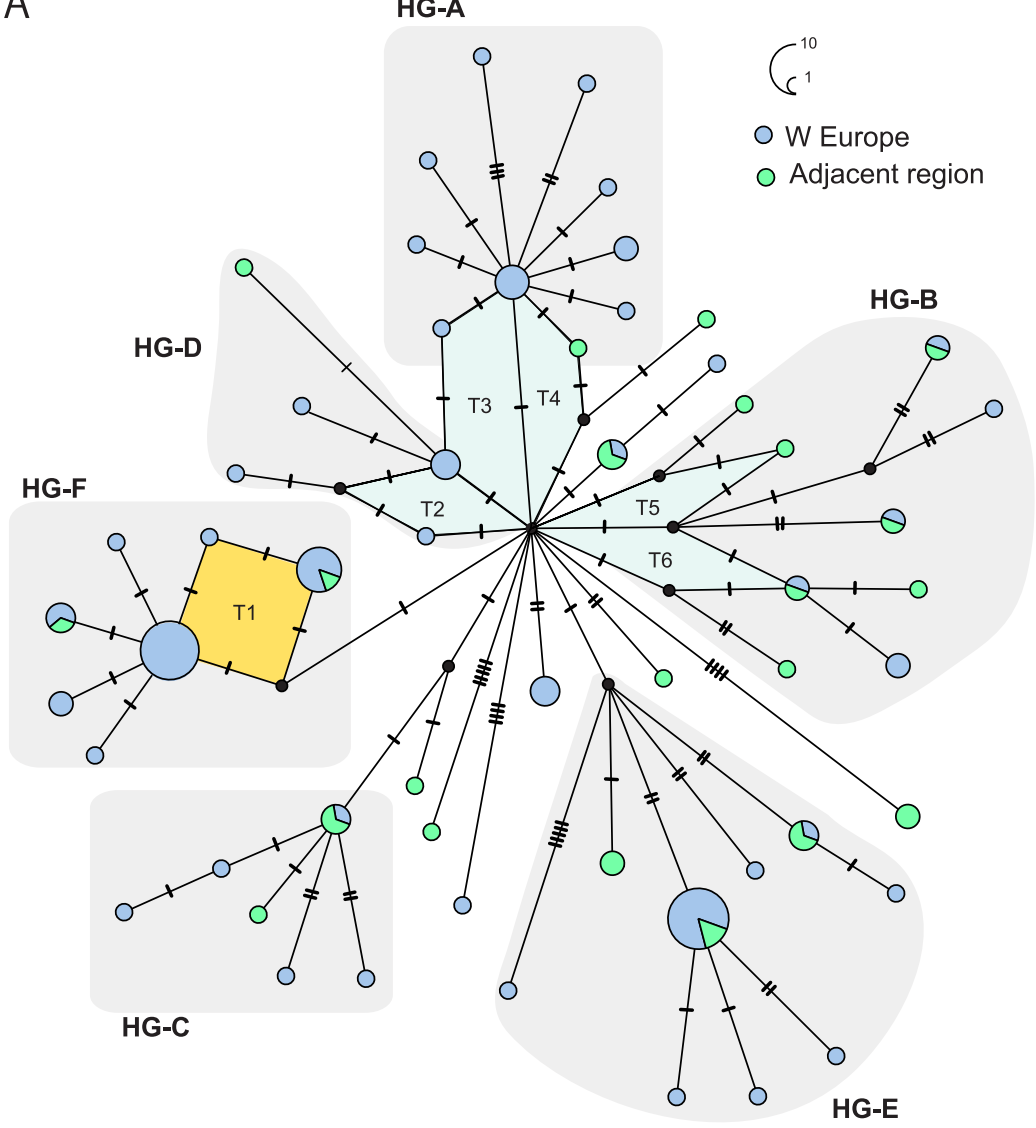

E

HG-F

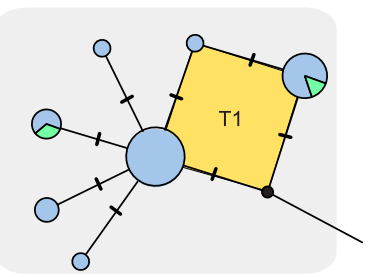

B

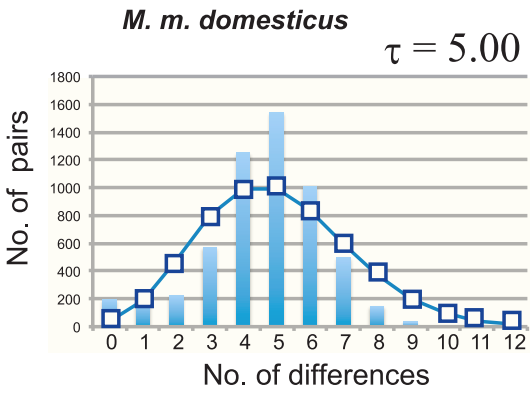

C

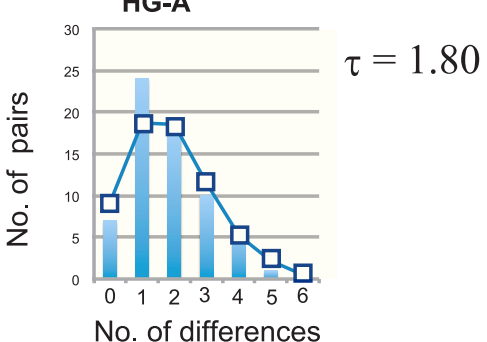

D

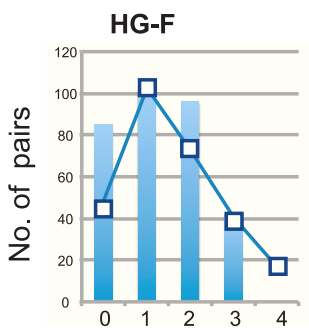

$\tau=1.60$

G

HG-F
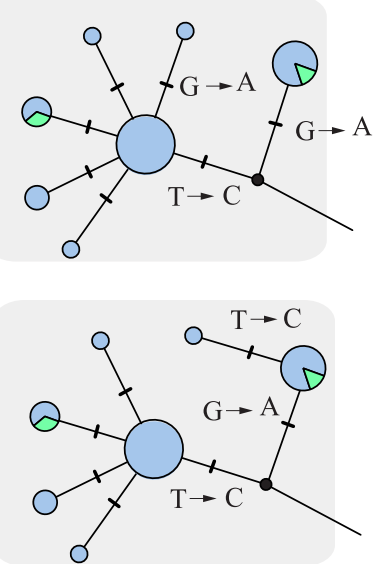

No. of differences

HG-F
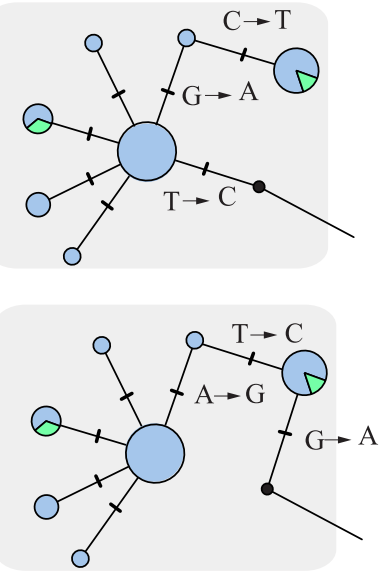

Figure 5. Median-joining networks constructed from (A) Cytb sequences ( $n=107 ; 958 \mathrm{bp}$ ) of Mus musculus domesticus. The number of mutations greater than ten between haplotypes is indicated on the branches. The size of the circles is proportional to the number of samples. The compositions of sample localities are also reflected in each haplotype. Six blocks (T1-T6) forming the 'torso' are indicated. Mismatch distributions of the mitochondrial Cytb sequences using data for Mus musculus (B), haplotype groups (HG, Fornuskova et al., 2014) HG-A (C), and HG-F (D) show significant evidence of expansion. Bars indicate the observed frequencies of mutations between haplotypes and the line denotes the expected frequency under the sudden expansion model. Haplogroup HG-F addresses structure T1 (E), which can be explained by either parallel mutation (F) or back (reverse) mutation $(\mathrm{G})$. 
The DOM cluster (Figure 5B) and haplogroups HG-A (Figure 5C) and HG-F (Figure 5D) were subjected to a mismatch distribution analysis and neutrality tests using Arlequin 3.5.1.2 (Excoffier, 2004; Excoffier and Lischer, 2010), confirming the notion of rapid overall expansion. The $\tau$ value of the DOM cluster $(\mathrm{n}=107)$ was $5.00(95 \%$ confidence interval (CI): 4.40-5.52) and the estimated time when the expansion started $(T)$ was 53900 (95\% CI: 474400-59600) years ago, with an evolutionary rate of 0.047 substitutions/ site/myr, attributing the expansion event to the abrupt warming in early MIS 3 . This is in good agreement with the estimate of Rajabi-Maham et al. (2008) of 60000 years ago for the DOM D-loop sequence data.

Haplogroup HG-A $(n=12)$ comprised haplotypes from western Europe and experienced a rapid expansion event, with a $\tau$ value of 1.82 (95\% CI: 0.95-3.00). With an evolutionary rate of 0.11 substitutions/site/myr, the calculated start of the expansion was 8400 (95\% CI: 4400-13800) years ago. By contrast, HG-F comprising haplotypes mainly from northwestern Europe had a $\tau$ value of 1.60 (95\% CI $0.57-3.06$ ), with an estimated expansion start time of 7600 (95\% CI: 2700-14500) years ago. Conceivably, the rapid population growth of the European house mouse is linked to the early introduction of agriculture to Europe roughly 7000-8000 years ago (Shennan et al., 2013).

\section{Application of the evolutionary rate to evolution- ary events in the Holocene}

Inference of the evolutionary history of the house mouse from whole mitochondrial genome sequences

We applied the time-dependent evolutionary rate to assess the natural history of the house mouse, addressing the factors shaping its long-distance dispersal with human agriculture in prehistoric times ( $\mathrm{Li}$ et al., 2020b). We determined whole genome sequences of $98 \mathrm{M}$. musculus collected mainly from Asia, constructed a phylogenetic tree, and estimated time using BEAST software (Drummond and Rambaut, 2007). Figure 6 shows a schematic representation of the early prehistoric eastward movements of two M. musculus subspecies, M. m. musculus (MUS) and M. m. castaneus (CAS), as predicted from the whole mitochondrial genome sequences ( $\mathrm{Li}$ et al., 2020b). The movement of MUS was achieved mainly by sublineage MUS-1 and involved five processes: (1) the initial spread of MUS-1 to northern Eurasia, including westernmost China; (2) movement to the eastern part of western China; (3) expansion to northern China; (4) introduction to the Korean peninsula; and (5) colonization of Japan by a descendant of the Korean haplotype group. The eastward movement of the CAS lineage was undertaken by sublineage CAS-1, resulting in coverage of northernmost China by 9000 years ago. The second CAS-1 dispersal event was movement either from the east coast of India to southern China or vice versa. The next step is simultaneous dispersal from southern China to several peripheral regions, including the eastern coast of India, Sri Lanka, and Bangladesh. The final step is dispersal from southern China to the Japanese archipelago, Far Eastern Russia, and Yunnan, China.

The history of the house mouse inferred from phylogenet- ic analysis with 0.11 substitutions/site/myr matches the spatiotemporal dynamics of prehistoric humans based on agriculture, particularly the introductions of millet farming in the Korean peninsula around 5300 years ago (Crawford and Lee, 2003; Li et al., 2020a) and paddy rice culture to the Japanese islands in the early Yayoi period 3000 years ago (Crawford and Lee, 2003; Fuller, 2011). The Austronesian language peoples dispersed to distant locations, including Indonesia, 4000-5000 years (e.g. Lipson et al., 2014; Deng et al., 2020) and this may have caused the dispersal of the house mouse to Indonesia. Recent work on the population dynamics of $M$. musculus from Myanmar showed evident population expansion in the $C y t b$ sequence data that began about 5400 (95\% CI: 2300-8000) years ago (Maung Maung Theint et al., 2020). In addition, the migration from southern China to the Japanese archipelago was deduced to have occurred 3500 years ago (Li et al., 2020b), which may be correlated with the introduction of cold-tolerant temperate japonica rice strains developed in response to the extreme environmental degradation 4200 years ago (Mao et al., 2019; Guo et al., 2020; Gutaker et al., 2020).

\section{A presumed evolutionary trend in the time- dependent evolutionary rate of mtDNA}

One concern is the mechanisms underlying the time dependency of the mtDNA evolutionary rate. A plausible hypothesis is that a weakly harmful mutation is removed over time (e.g. Rand, 2001; Penny, 2005; Henn et al., 2009; Ho et al., 2011), so the evolutionary rate will reflect the rate of weak deleterious mutations, in addition to the rate of neutral mutations that are not harmful (Figure 2). From this perspective, the initial stage (Phase I) is one in which new mutations are created, some of which are subjected to selection pressure and removed in the subsequent stage (Phase II). This probably occurs because, while plants and other organisms have a specific mtDNA repair system, animals lack this system (Wu et al., 2020). In the early stage of divergence in animal mtDNA, the rate of evolution is inevitably high because the proportion of mildly deleterious mutations is relatively high. As the divergence time becomes older, the proportion of the latter increases and the former portion becomes negligible.

The removal of weakly deleterious mutations is known as compensatory evolution and can be explained by the removal of the weakly deleterious mutations from each sequence (Meer et al., 2010; Osada, 2016). One of the mechanisms is called back (reverse) mutation. In relation to this issue, homoplasious mutations appear as a 'torso' in a network (Bandelt et al., 1999). The torso structure can be either explained by parallel or back mutations, because mtDNA does not experience recombination (Figure $5 \mathrm{E}-\mathrm{G}$ ).

The occurrence of homoplasious mtDNA mutations is well documented (Rand, 2001; Henn et al., 2009). This may due to their particular mode of evolution, namely a severe germline bottleneck (Rand, 2001; Zaidi et al., 2019), which may contribute to the relatively high frequency of homoplasy given the high incidence of competition among haplotypes during oocyte development. The trends to a high AT base composition, high transition-to-transversion ratio, high 


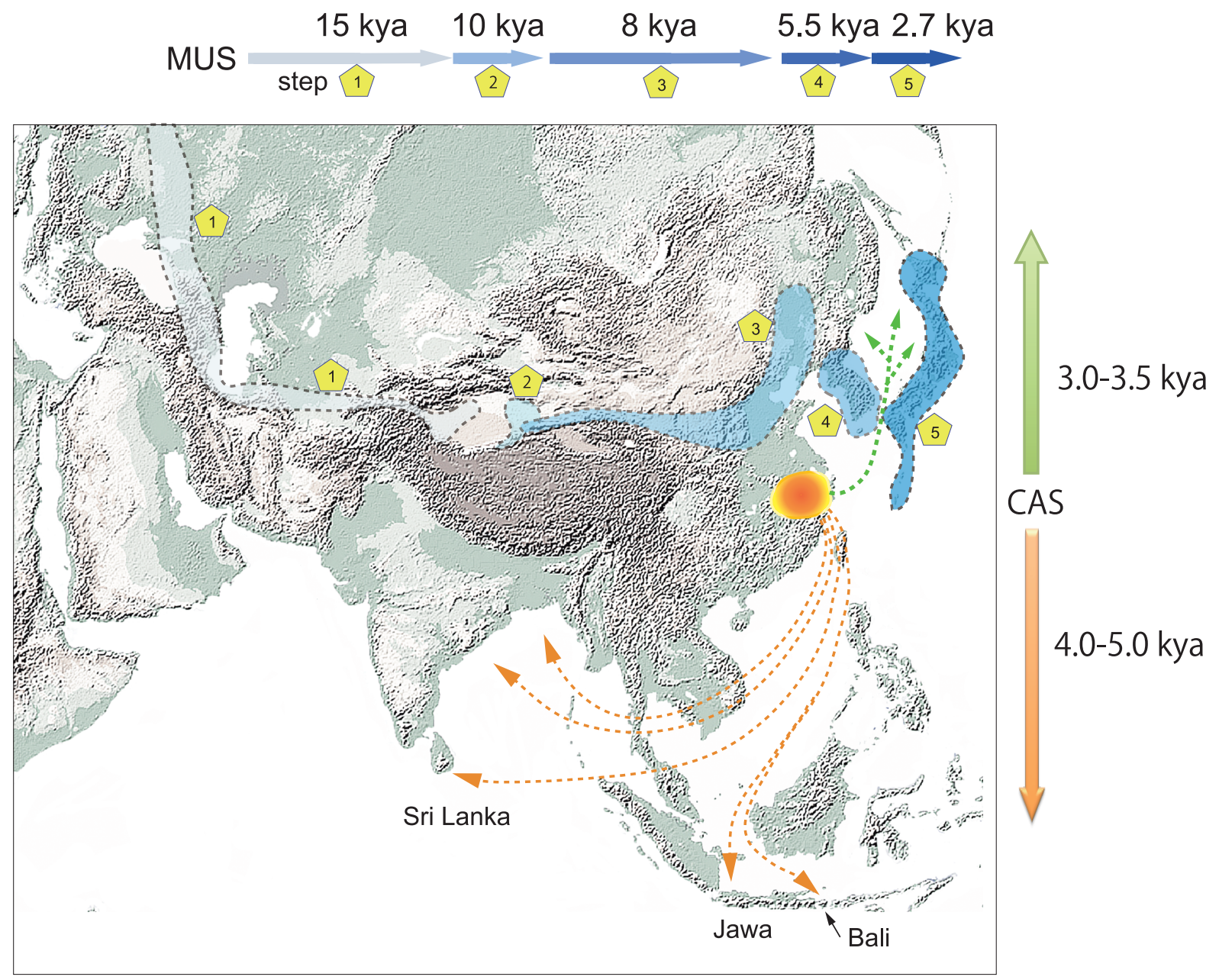

Figure 6. Schematic representation of the early prehistoric eastward movements of two subspecies of M. musculus, M. m. musculus (MUS) and M. m. castaneus (CAS), as predicted from the whole mitochondrial genome sequences $(\sim 16000 \mathrm{bp}$; Li et al., $2020 \mathrm{~b})$. The prehistoric introduction of MUS to East Asia is characterized as a stepwise eastward movement: (1) the initial spread to northern Eurasia, including westernmost China; (2) movement to the eastern part of western China; (3) expansion to northern China; (4) introduction to the Korean peninsula; and (5) colonization of Japan by a descendant of the Korean haplotype group. The initial eastward movement of CAS from its predicted homeland in the Middle East and India resulted in it covering northernmost China by 9000 years ago. The next step involves simultaneous dispersal from southern China to several peripheral regions, including the eastern coast of India, Sri Lanka, Bangladesh, and Indonesia. The final step is dispersal from southern China to the Japanese archipelago, Russian Far East, and Yunnan, China.

codon bias, and low non-synonymous/synonymous substitution rate ratio of mtDNA nucleotides mean that mtDNA evolves in a highly constrained manner (Rand, 2001); in this context, back-mutation in Phase II may explain all the evolutionary constraints well. If we address the mutations related to the torsos that may have emerged via back-mutation, it would provide useful clues to the mechanisms underlying the time dependency of mtDNA.

\section{Comparison of the time dependency among mtDNA genes}

Does the time dependency of mtDNA genes differ? Here, I examined the Cytb (1140 bp), COI (1463 bp), and ND2 (929 bp) genes from 98 house mouse whole genome sequences (16038 bp; Li et al., 2020b). I identified two phases of haplotype diversity with distinct genetic distances $(d)$ : one for divergence within the subspecies groups $(0<d<0.015)$, and the other for divergence between subspecies groups $(0.02<d<0.03)$. In the relatively shallow comparison of Cytb, COI, and ND2, the ratios to that of the whole mitochondrial genome sequences are 1.3, 1.1, and 1.0 , respectively (Figure $7 \mathrm{~A}-\mathrm{C}$ ), indicating that the three genes are somewhat similar. By contrast, on comparing the relatively deeper divergence, the ratios are 1.3, 0.96, and 1.45 , respectively (Figure 7D-F), and the differences among genes are larger, suggesting that the evolutionary rates are similar in the initial accumulation of mutations and differ with the removal of moderately deleterious mutations. In other words, the difference in evolutionary rates among gene regions is due to the difference in the extent of purifying selection among gene regions; the deleterious effects are greater in ND2 than Cytb. This provides evidence of the time dependency of the evolutionary rate of mtDNA. 
A

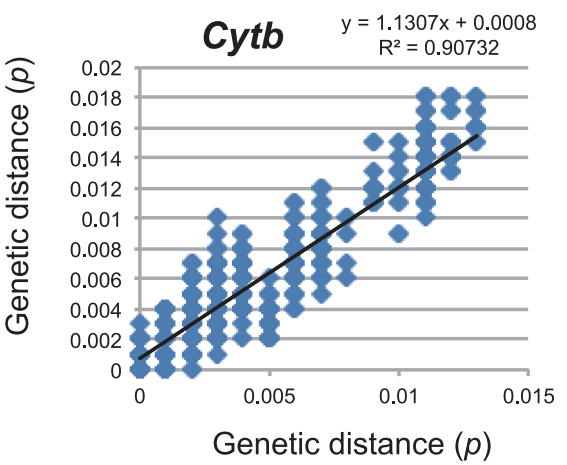

B

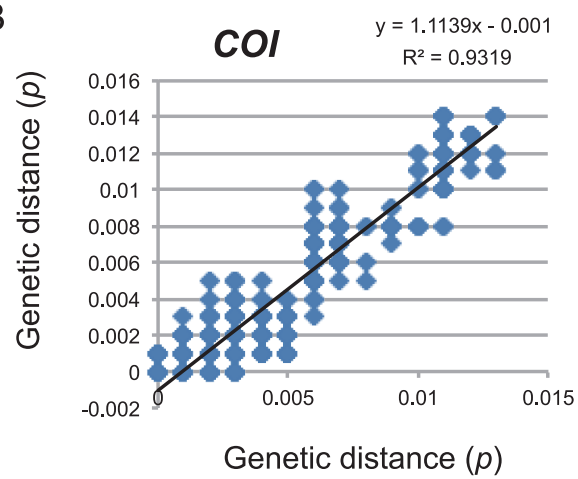

C

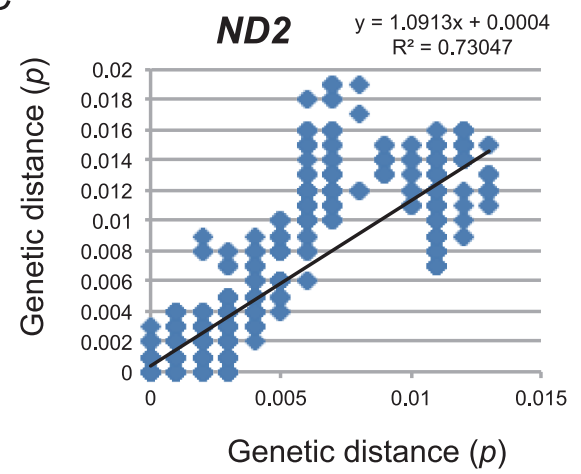

mitogenome
D

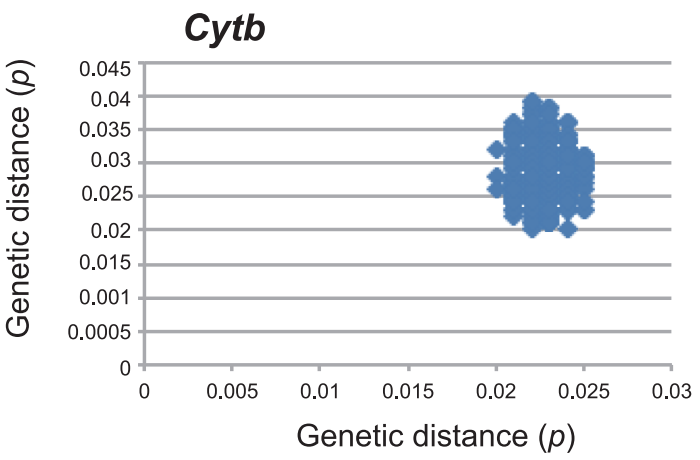

E

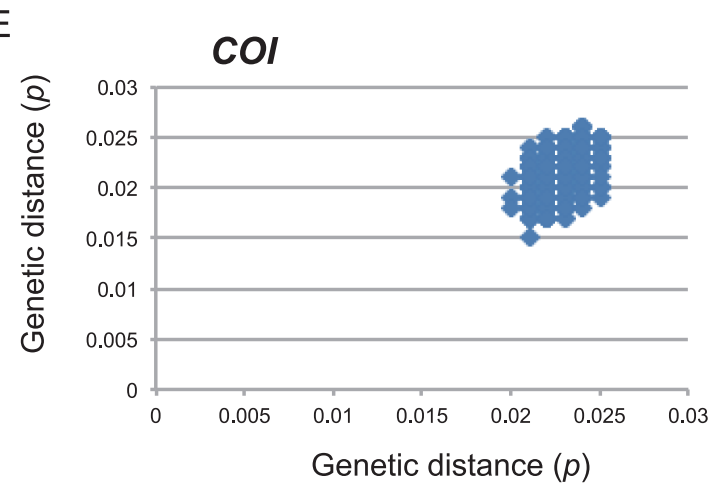

F

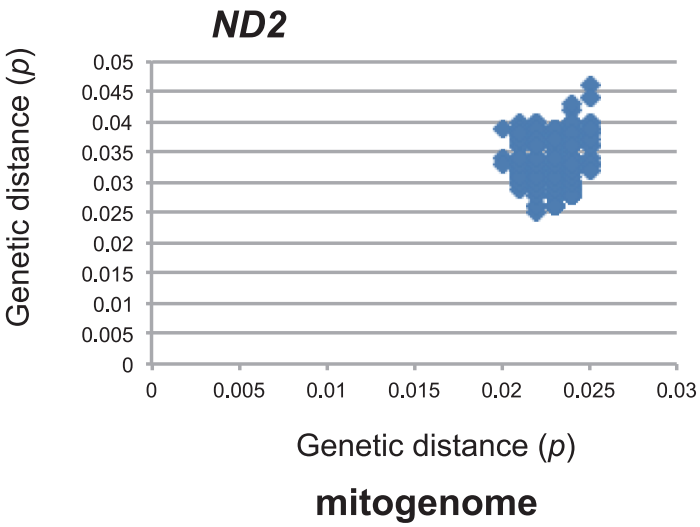

Figure 7. (A) Scatterplots showing the relationships between the genetic distances among each mitochondrial gene and the whole mitochondrial sequences. This plots the genetic distance (K2P) of the mitochondrial cytochrome $b$ (Cytb, $1140 \mathrm{bp})$ (A, D), cytochrome c oxidase I (COI, $1463 \mathrm{bp})(\mathrm{B}, \mathrm{E})$, and NADH dehydrogenase subunit 2 (ND2, $929 \mathrm{bp})$ genes $(\mathrm{C}, \mathrm{F})$ against the whole genome sequence $(16038 \mathrm{bp}) \mathrm{based}$ on 98 mouse mitogenome sequences (Li et al., 2020b). The plots compare the younger (A-C) and older (D-F) divergences, representing within- and between-subspecies divergence, respectively. The ratios are similar among the three protein-coding sequences for the younger divergences $(\sim 1.1)$, but differ for the deeper divergences $(1.28,0.96$, and 1.45 for $C y t b, C O I$, and $N D 2$, respectively) against whole mitochondrial sequences.

\section{Conclusion}

Considering the global environmental changes during the late Quaternary (i.e. the last 150000 years), the time periods responsible for the rapid expansion events were estimated to be around 11000, 15000, 53000, and 130000 years ago. These give evolutionary rates of $0.11,0.11,0.047$, and 0.029 substitutions/site/myr, respectively, showing apparent time dependency (Figure 3C). The evolutionary history of the house mouse M. musculus inferred from the higher evolutionary rate effectively explains its association with regional agricultural development in prehistoric times, implying that the rate is applicable for assessing divergence events in the Holocene. The higher evolutionary rate might apply to a short timescale, i.e. the last 20000 years; lengthening the timescale would give unrealistic results (Figure 3C). I would like to emphasize that the Japanese archipelago, which consists of many islands extending from subarctic to subtropical and separated by deep sea, makes it possible to utilize biogeographic calibration points to draw evolutionary rate curves in other faunal groups as well. 


\section{Acknowledgments}

I would like to thank Hiroki Oota for organizing this special issue. I wish to express my appreciation to Naoki Osada, Gohta Kinoshita, Alexey Kryukov, Jun Sato, and Naruya Saitou for giving constructive suggestions for improving this article. In particular, I would like to thank Yutaro Suzuki, Kaori Hanazaki, and Yuta Inoue for determining the major parts of our achievements regarding time-dependent evolutionary rates. Finally, I sincerely thank Kazuo Moriwaki and Kimiyuki Tsuchiya for their continuing support and encouragement. This study was supported by JSPS KAKENHI grant number JS18H05508.

\section{References}

Abramson N.I., Petrova T.V., Dokuchaev N.E., Obolenskaya E.V., and Lissovsky A.A. (2012) Phylogeography of the gray redbacked vole Craseomys rufocanus (Rodentia: Cricetidae) across the distribution range inferred from nonrecombining molecular markers. Russian Journal of Theriology, 11: 137156.

Aoki D., Kinoshita G., Kryukov A.P., Nishiumi I., Lee S., and Suzuki H. (2018) Quaternary-related genetic differentiation and parallel population dynamics of the Eurasian jay (Garrulus glandarius) in the circum-Japan Sea region. Journal of Ornithology, 159: 1087-1097.

Avise J.C. (1986) Mitochondrial DNA and the evolutionary genetics of higher animals. Philosophical Transactions of the Royal Society of London. B, Biological Sciences, 312: 325-342.

Bandelt H.J., Forster P., and Rohl A. (1999) Median-joining networks for inferring intraspecific phylogenies. Molecular, Biology and Evolution, 16: 37-48.

Bintanja R., van de Wal R.S.W., and Oerlemans J. (2005) Modelled atmospheric temperatures and global sea levels over the past million years. Nature, 437: 125-128.

Brown E.T., Johnson T.C., Scholz C.A., Cohen A.S., and King J.W. (2007) Abrupt change in tropical African climate linked to the bipolar seesaw over the past 55,000 years. Geophysical Research Letters, 34: L20702.

Brown W.M., George M., and Wilson A.C. (1979) Rapid evolution of animal mitochondrial DNA. Proceedings of the National Academy of Sciences of the United States of America, 76: 1967-1971.

Crawford G.W. and Lee G.A. (2003) Agricultural origins in the Korean Peninsula. Antiquity, 77: 87-95.

Deng Z., Hung H.C., Carson M.T., Oktaviana A.A., Hakim B., and Simanjuntak T. (2020) Validating earliest rice farming in the Indonesian Archipelago. Scientific Reports, 10: 10984

Drummond A.J. and Rambaut A. (2007) BEAST: Bayesian evolutionary analysis by sampling trees. BMC Evolutionary Biology, $7: 1-8$.

Excoffier L. (2004) Patterns of DNA sequence diversity and genetic structure after a range expansion: lessons from the infiniteisland model. Molecular Ecology, 13: 853-864.

Excoffier L. and Lischer H.E.L. (2010) Arlequin suite ver 3.5: a new series of programs to perform population genetics analyses under Linux and Windows. Molecular Ecology Resources, 10: $564-567$

Fickel J., Hauffe H.C., Pecchioli E., Soriguer R., Vapa L., and Pitra C. (2008) Cladogenesis of the European brown hare (Lepus europaeus Pallas, 1778). European Journal of Wildlife Research, 54: 495

Fornuskova A., Bryja J., Vinkler M., Macholán M., and Piálek J. (2014) Contrasting patterns of polymorphism and selection in bacterial-sensing toll-like receptor 4 in two house mouse subspecies. Ecology and Evolution, 14: 2931-2944.
Fuller D.Q. (2011) Pathways to Asian civilizations: tracing the origins and spread of rice and rice cultures. Rice, 4: 78-92.

García-Rodríguez O., Andreou D., Herman J.S., Mitsainas G.P., Searle J.B., et al. (2018) Cyprus as an ancient hub for house mice and humans. Journal of Biogeography, 45: 2619-2630.

Guo H., ZengY., Li J., Ma X., Zhang Z., Lou Q., et al. (2020) Differentiation, evolution and utilization of natural alleles for cold adaptability at the reproductive stage in rice. Plant Biotechnology Journal, 18: 2491-2503. https://doi.org/10.1111/ pbi.13424

Gutaker R.M., Groen S.C., Bellis E.S., Choi J.Y., Pires I.S., et al. (2020) Genomic history and ecology of the geographic spread of rice. Nature Plants, 6: 492-502.

Hanazaki K., Tomozawa M., Suzuki Y., Kinoshita G., Yamamoto M., Irino T., and Suzuki H. (2017) Estimation of evolutionary rates of mitochondrial DNA in two Japanese wood mouse species based on calibrations with Quaternary environmental changes. Zoological Science, 34: 201-210.

Harigai W., Saito A., Suzuki H., and Yamamoto M. (2020) Genetic diversity of Ligidium isopods in Hokkaido and Niigata, northern Japan, based on mitochondrial DNA analysis. Zoological Science, 37: 417-428. https://doi.org/10.2108/zs200017

Harrison S., Smith D.E., and Glasser N.F. (2019) Late Quaternary meltwater pulses and sea level change. Journal of Quaternary Science, 34: 1-15.

Helmens K.F. and Engels S. (2010) Ice-free conditions in eastern Fennoscandia during early Marine Isotope Stage 3: lacustrine records. Boreas, 39: 399-409.

Helmens K.F., Risberg J., Jansson K.N., Weckström J., Berntsson A., et al. (2009) Early MIS 3 glacial lake evolution, icemarginal retreat pattern and climate at Sokli (northeastern Fennoscandia). Quaternary Science Reviews, 28: 1880-1894.

Henn B.M., Gignoux C.R., Feldman M.W., and Mountain J.L. (2009) Characterizing the time dependency of human mitochondrial DNA mutation rate estimates. Molecular Biology and Evolution, 26: 217-230.

Herman J.S. and Searle J.B. (2011) Post-glacial partitioning of mitochondrial genetic variation in the field vole. Proceedings of the Royal Society B, Biological Sciences, 278: 3601-3607.

Herman J.S., Jóhannesdóttir F., Jones E.P., McDevitt A.D., Michaux J.R., et al. (2017) Post-glacial colonization of Europe by the wood mouse, Apodemus sylvaticus: evidence of a northern refugium and dispersal with humans. Biological Journal of the Linnean Society, 120: 313-332.

Hewitt G.M. (2004) Genetic consequences of climatic oscillations in the Quaternary. Philosophical Transactions of the Royal Society of London Series B, Biological Sciences, 359: 183195.

Ho S.Y.W. and Larson G. (2006) Molecular clocks: when times are a-changin.' Trends in Genetics, 22: 79-83.

Ho S.Y.W., Lanfear R., Bromham L., Phillips M.J., Soubrier J., et al. (2011) Time-dependent rates of molecular evolution. Molecular Ecology, 20: 3087-3101.

Honda A., Murakami S., Harada M., Tsuchiya K., Kinoshita G., and Suzuki H. (2019) Late Pleistocene climate change and population dynamics of Japanese Myodes voles inferred from mitochondrial cytochrome $b$ sequences. Journal of Mammalogy, 100: 1156-1168. https://doi.org/10.1093/jmammal/gyz093

Igarashi Y. and Oba T. (2006) Fluctuations in the East Asian monsoon over the last $144 \mathrm{ka}$ in the northwest Pacific based on a high-resolution pollen analysis of IMAGES core MD01-2421. Quaternary Science Reviews, 25: 1447-1459.

Igarashi Y., Naruse T., Yatagai S., and Danhara T. (2012) Vegetation and climate history since MIS 7 in the Kenbuchi Basin, northern Hokkaido: comparison between MIS 6/5e and MIS 2/1. Quaternary Research (Japan), 51: 175-191. (in Japanese with English Summary).

Irwin D.M., Kocher T.D., and Wilson A.C. (1991) Evolution of the cytochrome $b$ gene of mammals. Journal of Molecular Evolution, 32: 128-144. 
Japan Association for Quaternary Research (1987) Quaternary Maps of Japan. University of Tokyo Press, Tokyo.

Kohli B.A., Fedorov V.B., Waltari E., and Cook J.A. (2015) Phylogeography of a Holarctic rodent (Myodes rutilus): testing high latitude biogeographical hypotheses and the dynamics of range shifts. Journal of Biogeography, 42: 377-389.

Kryštufek B., Lužnik M., and Buzan E.V. (2012) Mitochondrial cytochrome $b$ sequences resolve the taxonomy of field mice (Apodemus) in the western Balkan refugium. Acta Theriologica, 57: 1-7.

Kuwayama T., Nunome M., Kinoshita G., Abe K., and Suzuki H. (2017) Heterogeneous genetic make-up of Japanese house mice (Mus musculus) created by multiple independent introductions and spatio-temporally diverse hybridization processes. Biological Journal of the Linnean Society, 122: 661-674.

Lambeck K., Rouby H., Purcell A., Sun Y.Y., and Sambridge M. (2014) Sea level and global ice volumes from the Last Glacial Maximum to the Holocene. Proceedings of the National Academy of Sciences of the United States of America, 111: 1529615303.

Li T., Ning C., Zhushchikhovskaya I.S., Hudson M.J., and Robbeets M. (2020a) Millet agriculture dispersed from northeast China to the Russian Far East: integrating archaeology, genetics, and linguistics. Archaeological Research in Asia, 22: 100177.

Li Y., Fujiwara K., Osada N., Kawai Y., Takada T., et al. (2020b) House mouse Mus musculus dispersal in East Eurasia inferred from 98 newly determined complete mitochondrial genome sequences. Heredity, https://doi.org/10.1038/s41437-02000364-y

Lipson M., Loh P.-R., Patterson N., Moorjani P., Ko Y.-C., et al. (2014) Reconstructing Austronesian population history in Island Southeast Asia. Nature Communications, 5: 4689.

Lisiecki L.E. and Raymo M.E. (2005) A Pliocene-Pleistocene stack of 57 globally distributed benthic $\delta^{18} \mathrm{O}$ record. Paleoceanography and Paleoclimatology, 20: PA1003. http://dx. doi.org/10.1029/2004PA001071

Mao D., Xin Y., Tan Y., Hu X., Bai J., et al. (2019) Natural variation in the HAN1 gene confers chilling tolerance in rice and allowed adaptation to a temperate climate. Proceedings of the National Academy of Sciences of the United States of America, 116: 3494-3501.

Maung Maung Theint S., Thwe T., Myat Myat Zaw K., Shimada T., Bawm S., et al. (2020) Late Quaternary environmental and human impacts on the mitochondrial DNA diversity of four commensal rodents in Myanmar. Journal of Mammalian Evolution. https://doi.org/10.1007/s10914-020-09519-4

Meer M.V., Kondrashov A.S., Artzy-Randrup Y., and Kondrashov F.A. (2010) Compensatory evolution in mitochondrial tRNAs navigates valleys of low fitness. Nature, 464: 279-282.

Michaux J.R., Magnanou E., Paradis E., Nieberding C., and Libois R. (2003) Mitochondrial phylogeography of the woodmouse (Apodemus sylvaticus) in the Western Palearctic region. Molecular Ecology, 12: 685-697.
Osada N. (2016) Compensatory Evolution. In: Kliman R.M. (ed.), Encyclopedia of Evolutionary Biology. Academic Press, Amsterdam, Vol. 1, pp. 329-333.

Oshida T., Masuda R., and Ikeda K. (2009) Phylogeography of the Japanese giant flying squirrel, Petaurista leucogenys (Rodentia: Sciuridae): implication of glacial refugia in an arboreal small mammal in the Japanese islands. Biological Journal of the Linnean Society, 98: 47-60.

Penny D. (2005) Relativity for molecular clocks. Nature, 436: 183-184.

Rajabi-Maham H., Orth A., and Bonhomme F. (2008) Phylogeography and postglacial expansion of Mus musculus domesticus inferred from mitochondrial DNA coalescent from Iran to Europe. Molecular Ecology, 17: 627-641.

Rand D.M. (2001) The units of selection on mitochondrial DNA. Annual Review of Ecology and Systematics, 32: 415-448.

Rogers A.R. and Harpending H. (1992) Population growth makes waves in the distribution of pairwise genetic differences. Molecular Biology and Evolution, 9: 552-569.

Shennan S., Downey S., Timpson A., Edinborough K., Colledge S., et al. (2013) Regional population collapse followed initial agriculture booms in mid-Holocene Europe. Nature Communications, 4: 2486.

Slatkin M. and Hudson R.R. (1991) Pairwise comparisons of mitochondrial DNA sequences in stable and exponentially growing populations. Genetics, 129: 555-562.

Suzuki H., Nunome M., Kinoshita G., Aplin K.P., Vogel P., et al. (2013) Evolutionary and dispersal history of Eurasian house mice Mus musculus clarified by more extensive geographic sampling of mitochondrial DNA. Heredity, 111: 375-390.

Suzuki Y., Tomozawa M., Koizumi Y., Tsuchiya K., and Suzuki H. (2015) Estimating the molecular evolutionary rates of mitochondrial genes referring to Quaternary ice age events with inferred population expansions and dispersals in Japanese Apodemus. BMC Evolutionary Biology, 15: 187.

Weber M., Scholz D., Schröder-Ritzrau A., Deininger M., Spötl C., et al. (2018) Evidence of warm and humid interstadials in central Europe during early MIS 3 revealed by a multi-proxy speleothem record. Quaternary Science Reviews, 200: 276286.

Wilson A.C., Cann R.L., Carr S.M., George M., Gyllensten U.B., et al. (1985) Mitochondrial DNA and two perspectives on evolutionary genetics. Biological Journal of the Linnean Society, 26: $375-400$.

Wu Z., Waneka G., Broz A.K., King C.R., and Sloan D.B. (2020) MSH1 is required for maintenance of the low mutation rates in plant mitochondrial and plastid genomes. Proceedings of the National Academy of Science of the United States of America, 117: 16448-16455.

Zaidi A.A., Wilton P.R., Su M.S.W., Paul I.M., Arbeithuber B., et al. (2019) Bottleneck and selection in the germline and maternal age influence transmission of mitochondrial DNA in human pedigrees. Proceedings of the National Academy of Sciences of the United States of America, 116: 25172-25178. 